\title{
Erratum
}

\section{Detection of amino acetonitrile in Sgr B2(N)}

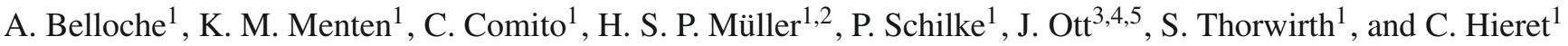 \\ 1 Max-Planck Institut für Radioastronomie, Auf dem Hügel 69, 53121 Bonn, Germany \\ e-mail: [belloche; kmenten; ccomito; schilke; sthorwirth; chieret] @mpifr-bonn.mpg.de \\ 2 I. Physikalisches Institut, Universität zu Köln, Zülpicher Str. 77, 50937 Köln, Germany \\ e-mail: hspm@ph1.uni-koeln.de \\ 3 National Radio Astronomy Observatory, 520 Edgemont Road, Charlottesville, VA 22903-2475, USA \\ e-mail: jott@nrao.edu \\ ${ }^{4}$ California Institute of Technology, 1200 E. California Blvd., Caltech Astronomy, 105-24, Pasadena, CA 91125-2400, USA \\ 5 CSIRO Australia Telescope National Facility, Cnr Vimiera \& Pembroke Roads, Marsfield NSW 2122, Australia
}

A\&A 482, 179-196 (2008), DOI: 10.1051/0004-6361:20079203

\section{ABSTRACT}

\begin{abstract}
Aims. A small error was recently found in the program used to compute the integrated intensities in the article Belloche et al. (2008, A\&A, 482, 179).

Methods. We provide new versions of Fig. 2 and Tables 3, 5, and 7 with the correct integrated intensities.

Results. The conclusions drawn by Belloche et al. (2008) are not significantly affected by these corrections. Only the mass, density, and central $\mathrm{H}_{2}$ column density of the compact source emitting the amino acetonitrile lines appear to have been underestimated by $30-40 \%$. This led to an overestimate of the abundance of amino acetonitrile by the same amount. However, the magnitude of this correction is less than the estimated uncertainties on these parameters.
\end{abstract}

Key words. astrobiology - astrochemistry - line: identification - stars: formation - ISM: individual objects: Sagittarius B2 errata, addenda

We found recently that some of the integrated intensities published in Belloche et al. (2008) were computed with a program containing a small error in the conversion from the frequency domain to the velocity domain. This error affects only spectra that cover a wide frequency range, i.e. the wideband single-dish $3 \mathrm{~mm}$ and $1 \mathrm{~mm}$ spectra. As a result, some of the intensities listed in Col. 10 of Table 3, some of the intensities used in Fig. 2, and all single-dish fluxes listed in Col. 13 of Tables 5 and 7 are incorrect (up to 40\%). These intensities have to be multiplied by $115.750 / v(\mathrm{GHz})$ for $v<116 \mathrm{GHz}$ and by $254.176 / v(\mathrm{GHz})$ for $v>200 \mathrm{GHz}$, with $v$ the central frequency of the integration range. The intensities of lines at other frequencies are not affected. We present in this erratum new versions of Fig. 2 and Tables 3, 5, and 7 corrected for this error.

The changes in Fig. 2 are not significant enough to change any of the conclusions we draw from the analysis of the singledish spectrum because our fitting method was not based on these integrated intensities. Therefore our best fit model is not affected.

The single-dish integrated fluxes of Table 5 are all $40 \%$ larger than before. Although this might suggest more extended emission than we initially thought, we actually think this increase does not change our conclusions for the following reason. The transition of cyanoacetylene in the vibrationally excited $v_{4}=$ 1 state at $81.77 \mathrm{GHz}$ is at such high energy $\left(E_{\mathrm{u}} / k_{\mathrm{B}}=1283 \mathrm{~K}\right)$ that it must come from one or several hot, very compact regions. We can exclude that several compact sources contribute to the single-dish flux since only one source is detected in the PdBI map (see Fig. 5j). Since our model does not show any

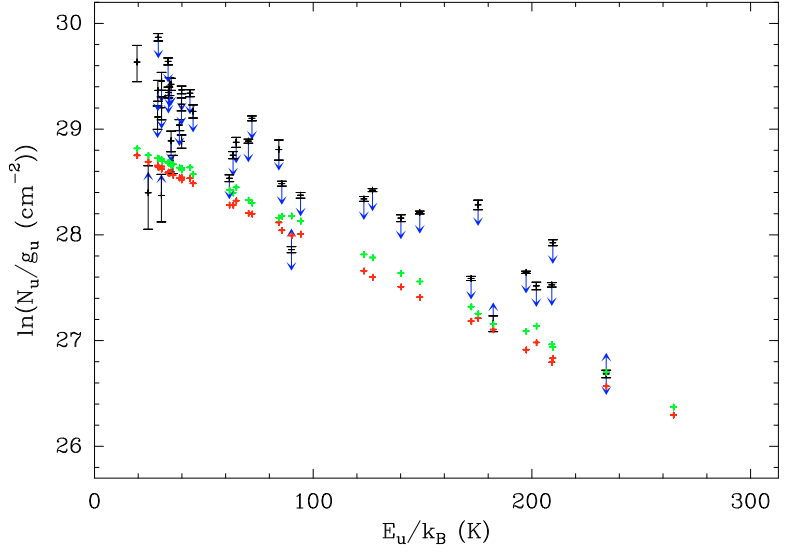

Fig. 2. Population diagram of amino acetonitrile in Sgr B2(N). The red points were computed in the optically thin approximation using the integrated intensities of our best-fit model of amino acetonitrile, while the green points were corrected for the opacity. The black points were computed in the optically thin approximation using the integrated intensities of the spectrum observed with the IRAM $30 \mathrm{~m}$ telescope. The error bars are $1 \sigma$ uncertainties on $N_{\mathrm{u}} / g_{\mathrm{u}}$. Blue arrows pointing downwards mark the transitions blended with transitions from other molecules, while blue arrows pointing upwards indicate that the baseline removed in the observed spectrum is uncertain. The arrow length is arbitrary. The measurement corresponding to feature 43 (at $E_{\mathrm{u}} / k_{\mathrm{B}}=265 \mathrm{~K}$ ) is not shown since the integrated intensity measured toward Sgr B2(N) is negative, due to the blend with $\mathrm{CN}$ absorption lines. 
Table 3. Transitions of amino acetonitrile detected toward Sgr B2(N) with the IRAM $30 \mathrm{~m}$ telescope.

\begin{tabular}{|c|c|c|c|c|c|c|c|c|c|c|c|c|}
\hline (1) & Transition & $\begin{array}{c}\text { Frequency } \\
(\mathrm{MHz}) \\
(3)\end{array}$ & $\begin{array}{c}\text { Unc. }^{b} \\
(\mathrm{kHz}) \\
(4)\end{array}$ & $\begin{array}{l}E_{1}^{c} \\
(\mathrm{~K}) \\
(5)\end{array}$ & $\begin{array}{l}\mathrm{S} \mu^{2} \\
\left(\mathrm{D}^{2}\right) \\
(6)\end{array}$ & $\begin{array}{c}\sigma^{d} \\
(\mathrm{mK}) \\
(7)\end{array}$ & (8) & (9) & $\begin{array}{c}I_{\mathrm{obs}}^{g} \\
\left(\mathrm{~K} \mathrm{~km} \mathrm{~s}^{-1}\right) \\
(10)\end{array}$ & $\begin{array}{c}I_{\mathrm{AAN}}^{g} \\
\left(\mathrm{~K} \mathrm{~km} \mathrm{~s}^{-1}\right) \\
(11)\end{array}$ & $\begin{array}{c}I_{\text {all }}^{g} \\
\left(\mathrm{~K} \mathrm{~km} \mathrm{~s}^{-1}\right) \\
(12)\end{array}$ & $\begin{array}{l}\text { Comments } \\
\text { (13) }\end{array}$ \\
\hline 1 & $9_{0,9}-8_{0,8}$ & 80947.479 & 7 & 16 & 60 & 33 & 1 & 0.13 & $0.93(16)$ & 0.38 & 0.42 & no blend \\
\hline 3 & $9_{5,5}-8_{5,4}$ & 81700.966 & 6 & 47 & 41 & 13 & 2 & 0.16 & $1.30(07)$ & 0.67 & 0.75 & partial blend with U-line \\
\hline 4 & $9_{5,4}-85,3$ & 81700.967 & 6 & 47 & 41 & 13 & 2 & - & - & - & - & - \\
\hline 5 & $9_{6,3}-8_{6,2}$ & 81702.498 & 5 & 60 & 33 & 13 & 2 & - & - & - & - & - \\
\hline 6 & $9_{6,4}-8_{6,3}$ & 81702.498 & 5 & 60 & 33 & 13 & 2 & - & - & - & - & - \\
\hline 7 & $9_{4,6}-8_{4,5}$ & 81709.838 & 6 & 35 & 48 & 13 & 3 & 0.23 & $0.55(06)$ & 0.66 & 0.73 & no blend \\
\hline 8 & $9_{7,2}-8_{7,1}$ & 81709.848 & 6 & 76 & 24 & 13 & 3 & - & - & - & - & - \\
\hline 9 & $9_{7,3}-8_{7,2}$ & 81709.848 & 6 & 76 & 24 & 13 & 3 & - & - & - & - & - \\
\hline 10 & $9_{4,5}-8_{4,4}$ & 81710.098 & 6 & 35 & 48 & 13 & 3 & - & - & - & - & - \\
\hline 11 & $9_{3,7}-8_{3,6}$ & 81733.892 & 6 & 27 & 53 & 13 & 4 & 0.11 & $0.71(06)$ & 0.32 & 1.46 & $\begin{array}{l}\text { blend with } \mathrm{CH}_{3} \mathrm{OCH}_{3} \text { and } \\
\mathrm{HCC}^{13} \mathrm{CN}, v_{6}=1\end{array}$ \\
\hline 12 & $9_{3,6}-8_{3,5}$ & 81756.174 & 6 & 27 & 53 & 13 & 5 & 0.11 & $0.55(06)$ & 0.32 & 0.32 & blend with U-line \\
\hline 13 & $9_{2,7}-8_{2,6}$ & 82224.644 & 7 & 21 & 57 & 19 & 6 & 0.12 & $0.27(08)$ & 0.36 & 0.35 & uncertain baseline \\
\hline 17 & $10_{2,9}-9_{2,8}$ & 90561.332 & 6 & 25 & 64 & 20 & 7 & 0.14 & $0.82(09)$ & 0.52 & 1.01 & $\begin{array}{l}\text { blend with weak } \\
\mathrm{C}_{2} \mathrm{H}_{5} \mathrm{CN}, v_{13}=1 / v_{21}=1\end{array}$ \\
\hline 18 & $10_{6,4}-96,3$ & 90783.538 & 6 & 64 & 43 & 14 & 8 & 0.28 & $1.96(06)$ & 1.05 & 1.40 & $\begin{array}{l}\text { partial blend with } \mathrm{CH}_{2}(\mathrm{OH}) \mathrm{CHO} \text { and } \\
\text { U-line }\end{array}$ \\
\hline 19 & $10_{6,5}-9_{6,4}$ & 90783.538 & 6 & 64 & 43 & 14 & 8 & - & - & - & - & - \\
\hline 20 & $10_{5,6}-9_{5,5}$ & 90784.281 & 6 & 50 & 50 & 14 & 8 & - & - & - & - & - \\
\hline 21 & $10_{5,5}-9_{5,4}$ & 90784.285 & 6 & 50 & 50 & 14 & 8 & - & - & - & - & - \\
\hline 22 & $10_{7,3}-9_{7,2}$ & 90790.259 & 6 & 80 & 34 & 14 & 9 & 0.09 & $0.65(06)$ & 0.32 & 0.56 & blend with U-line \\
\hline 23 & $10_{7,4}-9_{7,3}$ & 90790.259 & 6 & 80 & 34 & 14 & 9 & - & - & - & - & - \\
\hline 24 & $10_{4,7}-94,6$ & 90798.685 & 6 & 39 & 56 & 14 & 10 & 0.21 & $1.82(06)$ & 0.81 & 0.95 & blend with U-line \\
\hline 25 & $10_{4,6}-9_{4,5}$ & 90799.249 & 6 & 39 & 56 & 14 & 10 & - & - & - & - & - \\
\hline 28 & $10_{3,8}-9_{3,7}$ & 90829.945 & 6 & 31 & 60 & 14 & 11 & 0.13 & $1.07(06)$ & 0.47 & 0.51 & blend with U-line also in M? \\
\hline 29 & $10_{3,7}-9,3$ & 90868.038 & 6 & 31 & 60 & 14 & 12 & 0.13 & $0.63(06)$ & 0.47 & 0.57 & partial blend with U-line \\
\hline 30 & $10_{2,8}-9_{2,7}$ & 91496.108 & 8 & 25 & 64 & 24 & 13 & 0.15 & $1.09(11)$ & 0.53 & 0.71 & $\begin{array}{l}\text { partial blend with } \mathrm{CH}_{3} \mathrm{CN}, v_{4}=1 \text { and } \\
\text { U-line }\end{array}$ \\
\hline 32 & $11_{1,11}-10_{1,10}$ & 97015.224 & 8 & 25 & 72 & 21 & 14 & 0.18 & $2.44(09)$ & 0.71 & 1.78 & $\begin{array}{l}\text { partial blend with } \mathrm{C}_{2} \mathrm{H}_{5} \mathrm{OH} \text { and } \\
\mathrm{CH}_{3} \mathrm{OCHO}\end{array}$ \\
\hline 47 & $11_{3,9}-10_{3,8}$ & 99928.886 & 6 & 35 & 68 & 14 & 15 & 0.15 & $1.51(06)$ & 0.66 & 1.24 & partial blend with $\mathrm{NH}_{2} \mathrm{CN}$ and $\mathrm{U}$-line \\
\hline 48 & $11_{3,8}-10_{3,7}$ & 99990.567 & 7 & 35 & 68 & 14 & 16 & 0.15 & $0.93(06)$ & 0.66 & 0.74 & no blend \\
\hline 49 & $11_{2,9}-10_{2,8}$ & 100800.876 & 8 & 29 & 71 & 20 & 17 & 0.17 & $1.58(08)$ & 0.75 & 1.25 & $\begin{array}{l}\text { partial blend with } \mathrm{CH}_{3} \mathrm{CH}_{3} \mathrm{CO}, v=0 \\
\text { and U-line }\end{array}$ \\
\hline 50 & $11_{1,10}-10_{1,9}$ & 101899.795 & 8 & 26 & 72 & 34 & 18 & 0.18 & $0.63(14)$ & 0.81 & 0.88 & uncertain baseline \\
\hline 51 & $12_{1,12}-11_{1,11}$ & 105777.991 & 8 & 29 & 79 & 43 & 19 & 0.20 & $2.17(18)$ & 0.95 & 2.88 & $\begin{array}{l}\text { blend with } \mathrm{c}-\mathrm{C}_{2} \mathrm{H}_{4} \mathrm{O} \text { and } \\
\mathrm{C}_{2} \mathrm{H}_{5} \mathrm{CN}, v=0\end{array}$ \\
\hline 52 & $12_{0,12}-11_{0,11}$ & 107283.142 & 8 & 29 & 80 & 24 & 20 & 0.21 & $2.88(10)$ & 1.00 & 2.01 & blend with $\mathrm{C}_{2} \mathrm{H}_{5} \mathrm{OH}$ and U-line \\
\hline 53 & $12_{2,11}-11_{2,10}$ & 108581.408 & 7 & 34 & 77 & 20 & 21 & 0.19 & $1.59(08)$ & 0.97 & 1.93 & weak blend with $\mathrm{C}_{2} \mathrm{H}_{5} \mathrm{OH}$ \\
\hline 58 & $12_{5,8}-11_{5,7}$ & 108956.206 & 6 & 60 & 66 & 29 & 22 & 0.26 & $2.33(11)$ & 1.34 & 3.44 & blend with $\mathrm{C}_{2} \mathrm{H}_{5} \mathrm{OH}$ \\
\hline 59 & $12_{5,7}-11_{5,6}$ & 108956.229 & 6 & 60 & 66 & 29 & 22 & - & - & - & - & - \\
\hline 68 & $12_{3,10}-11_{3,9}$ & 109030.225 & 6 & 40 & 75 & 29 & 23 & 0.18 & $1.77(11)$ & 0.89 & 1.24 & $\begin{array}{l}\text { partial blend with } \mathrm{HC}_{3} \mathrm{~N}, v_{4}=1 \text {, } \\
\mathrm{C}_{2} \mathrm{H}_{5} \mathrm{OH} \text {, and U-line }\end{array}$ \\
\hline 71 & $12_{1,11}-11_{1,10}$ & 111076.901 & 8 & 31 & 79 & 25 & 24 & 0.21 & $1.20(10)$ & 1.08 & 1.39 & slightly shifted? \\
\hline 72 & $13_{1,13}-12_{1,12}$ & 114528.654 & 8 & 34 & 86 & 37 & 25 & 0.23 & $2.52(15)$ & 1.23 & 1.42 & partial blend with U-line \\
\hline 84 & $15_{10,5}-14_{10,4}$ & 136248.969 & 10 & 169 & 55 & 28 & 26 & 0.09 & $2.10(10)$ & 0.72 & 1.03 & blend with U-line \\
\hline 85 & $15_{10,6}-14_{10,5}$ & 136248.969 & 10 & 169 & 55 & 28 & 26 & - & - & - & - & - \\
\hline 89 & $15_{4,11}-14_{4,10}$ & 136303.599 & 6 & 65 & 93 & 28 & 27 & 0.21 & $3.99(09)$ & 1.61 & 4.02 & blend with $\mathrm{a}\left(\mathrm{CH}_{2} \mathrm{OH}\right)_{2}$ and $\mathrm{CH}_{3} \mathrm{C}_{3} \mathrm{~N}$ \\
\hline 92 & $15_{3,13}-14_{3,12}$ & 136341.155 & 6 & 57 & 96 & 28 & 28 & 0.24 & $2.91(10)$ & 1.81 & 2.22 & partial blend with U-line also in M \\
\hline 103 & $16_{5,12}-15_{5,11}$ & 145325.871 & 30 & 83 & 96 & 25 & 29 & 0.39 & $2.89(08)$ & 3.30 & 4.79 & $\begin{array}{l}\text { uncertain baseline, partial blend } \\
\text { with } \mathrm{C}_{2} \mathrm{H}_{5} \mathrm{CN}, v_{13}=1 / v_{21}=1\end{array}$ \\
\hline 104 & $16_{5,11}-15_{5,10}$ & 145326.209 & 30 & 83 & 96 & 25 & 29 & - & - & - & - & - \\
\hline 105 & $16_{10,6}-15_{10,5}$ & 145330.985 & 40 & 175 & 65 & 25 & 30 & 0.11 & $0.97(07)$ & 0.92 & 1.02 & uncertain baseline \\
\hline 106 & $16_{10,7}-15_{10,6}$ & 145330.985 & 40 & 175 & 65 & 25 & 30 & - & - & - & - & - \\
\hline 115 & $16_{3,14}-15_{3,13}$ & 145443.850 & 30 & 63 & 103 & 25 & 31 & 0.25 & $4.32(08)$ & 2.18 & 4.67 & $\begin{array}{l}\text { blend with } \mathrm{C}_{2} \mathrm{H}_{5} \mathrm{CN}, v=0 \text { and } \\
\text { U-line }\end{array}$ \\
\hline 118 & $16_{1,15}-15_{1,14}$ & 147495.789 & 6 & 55 & 106 & 31 & 32 & 0.29 & $3.27(11)$ & 2.54 & 11.47 & partial blend with $\mathrm{H}_{3} \mathrm{C}^{13} \mathrm{CN}, v_{8}=1$ \\
\hline 139 & $17_{4,13}-16_{4,12}$ & 154542.406 & 5 & 79 & 107 & 112 & 33 & 0.44 & $13.24(42)$ & 4.63 & 5.52 & blend with U-line \\
\hline 140 & $17_{3,15}-16_{3,14}$ & 154544.046 & 5 & 70 & 109 & 112 & 33 & - & - & - & - & - \\
\hline 145 & $18_{7,12}-17_{7,11}$ & 163454.794 & 5 & 127 & 101 & 38 & 34 & 0.49 & $10.38(13)$ & 5.32 & 16.48 & $\begin{array}{l}\text { partial blend with } \mathrm{HC}^{13} \mathrm{CCN} \text {, } \\
v_{6}=1 \text { and } \mathrm{HCC}^{13} \mathrm{CN}, v_{6}=1\end{array}$ \\
\hline 146 & $18_{7,11}-17_{7,10}$ & 163454.794 & 5 & 127 & 101 & 38 & 34 & - & - & - & - & - \\
\hline 147 & $18_{8,10}-17_{8,9}$ & 163456.136 & 6 & 146 & 96 & 38 & 34 & - & - & - & - & - \\
\hline 148 & $18_{8,11}-17_{8,10}$ & 163456.136 & 6 & 146 & 96 & 38 & 34 & - & - & - & - & - \\
\hline
\end{tabular}


Table 3. continued.

\begin{tabular}{|c|c|c|c|c|c|c|c|c|c|c|c|c|}
\hline (1) & Transition & $\begin{array}{l}\text { Frequency } \\
\text { (MHz) } \\
\text { (3) }\end{array}$ & $\begin{array}{l}\text { Unc. }^{b} \\
(\mathrm{kHz}) \\
(4)\end{array}$ & $\begin{array}{l}E_{1}^{c} \\
(\mathrm{~K}) \\
(5)\end{array}$ & $\begin{array}{l}\mathrm{S} \mu^{2} \\
\left(\mathrm{D}^{2}\right) \\
(6)\end{array}$ & $\begin{array}{c}\sigma^{d} \\
(\mathrm{mK}) \\
(7)\end{array}$ & $F^{e}$ & (9) & $\begin{array}{c}I_{\mathrm{obs}}^{g} \\
\left(\mathrm{~K} \mathrm{~km} \mathrm{~s}{ }^{-1}\right) \\
(10)\end{array}$ & $\begin{array}{c}I_{\mathrm{AAN}}^{g} \\
\left(\mathrm{~K} \mathrm{~km} \mathrm{~s}^{-1}\right) \\
(11)\end{array}$ & $\begin{array}{c}I_{\text {all }}^{g} \\
\left(\mathrm{~K} \mathrm{~km} \mathrm{~s}^{-1}\right) \\
(12)\end{array}$ & $\begin{array}{l}\text { Comments } \\
\text { (13) }\end{array}$ \\
\hline 149 & $18_{9,9}-17_{9,8}$ & 163470.472 & 8 & 166 & 90 & 38 & 35 & 0.41 & $15.17(14)$ & 5.57 & 21.97 & partial blend with $\mathrm{HCC}^{13} \mathrm{CN}, v_{7}=1$ \\
\hline 150 & $18_{9,10}-17_{9,9}$ & 163470.472 & 8 & 166 & 90 & 38 & 35 & - & - & - & - & - \\
\hline 151 & $18_{6,13}-17_{6,12}$ & 163473.305 & 5 & 111 & 106 & 38 & 35 & - & - & - & - & - \\
\hline 152 & $18_{6,12}-17_{6,11}$ & 163473.321 & 5 & 111 & 106 & 38 & 35 & - & - & - & - & - \\
\hline 155 & $18_{11,7}-17_{11,6}$ & 163525.533 & 11 & 216 & 75 & 38 & 36 & 0.49 & $10.26(13)$ & 5.27 & 17.96 & blend with $\mathrm{HC}_{3} \mathrm{~N}, v_{4}=1$ \\
\hline 156 & $18_{11,8}-17_{11,7}$ & 163525.533 & 11 & 216 & 75 & 38 & 36 & - & - & - & - & - \\
\hline 157 & $18_{5,14}-17_{5,13}$ & 163526.183 & 4 & 97 & 110 & 38 & 36 & - & - & - & - & - \\
\hline 158 & $18_{5,13}-17_{5,12}$ & 163527.171 & 4 & 97 & 110 & 38 & 36 & - & - & - & - & - \\
\hline 163 & $18_{4,15}-17_{4,14}$ & 163635.326 & 5 & 86 & 114 & 38 & 37 & 0.25 & $4.07(11)$ & 2.82 & 5.01 & partial blend with $\mathrm{C}_{3} \mathrm{H}_{7} \mathrm{CN}$ \\
\hline 164 & $18_{3,16}-17_{3,15}$ & 163640.468 & 5 & 78 & 116 & 38 & 38 & 0.28 & $4.65(11)$ & 2.98 & 6.77 & partial blend with $\mathrm{C}_{3} \mathrm{H}_{7} \mathrm{CN}$ \\
\hline 177 & $19_{6,14}-18_{6,13}$ & 172566.092 & 50 & 119 & 114 & 44 & 39 & 0.38 & $10.01(14)$ & 4.39 & 6.43 & $\begin{array}{l}\text { partial blend with U-line and } \\
\mathrm{HCC}^{13} \mathrm{CN}, v_{7}=1\end{array}$ \\
\hline 178 & $19_{6,13}-18_{6,12}$ & 172566.092 & 50 & 119 & 114 & 44 & 39 & - & - & - & - & - \\
\hline 227 & $23_{4,20}-22_{4,19}$ & 209272.189 & 6 & 130 & 148 & 58 & 40 & 0.26 & $8.85(29)$ & 4.62 & 14.85 & blend $\mathrm{CH}_{3} \mathrm{CH}_{3} \mathrm{CO}, v=0$ \\
\hline 237 & $23_{1,22}-22_{1,21}$ & 209629.913 & 9 & 113 & 152 & 45 & 41 & 0.32 & $10.95(24)$ & 5.53 & 30.87 & $\begin{array}{l}\text { blend with } \mathrm{HC}^{13} \mathrm{CCN}, v_{7}=2 \text { and } \\
\mathrm{HCC}^{13} \mathrm{CN}, v_{7}=2\end{array}$ \\
\hline 247 & $25_{9,16}-24_{9,15}$ & 227040.487 & 50 & 230 & 145 & 96 & 42 & 0.29 & $10.72(55)$ & 9.45 & 35.32 & $\begin{array}{l}\text { partial blend with } \mathrm{CN} \text { absorption } \\
\text { and } \mathrm{CH}_{3} \mathrm{CH}_{3} \mathrm{CO}, v_{\mathrm{t}}=1\end{array}$ \\
\hline 248 & $25_{9,17}-24_{9,16}$ & 227040.487 & 50 & 230 & 145 & 96 & 42 & - & - & - & - & - \\
\hline 249 & $25_{8,18}-24_{8,17}$ & 227045.287 & 50 & 210 & 149 & 96 & 42 & - & - & - & - & - \\
\hline 250 & $25_{8,17}-24_{8,16}$ & 227045.287 & 50 & 210 & 149 & 96 & 42 & - & - & - & - & - \\
\hline 251 & $25_{10,15}-24_{10,14}$ & 227055.944 & 50 & 254 & 139 & 96 & 43 & 0.15 & $-0.72(44)$ & 3.29 & 3.62 & partial blend with $\mathrm{CN}$ absorption \\
\hline 252 & $25_{10,16}-24_{10,15}$ & 227055.944 & 50 & 254 & 139 & 96 & 43 & - & - & - & - & - \\
\hline 253 & $25_{7,19}-24_{7,18}$ & 227079.847 & 50 & 191 & 153 & 96 & 44 & 0.32 & $12.25(44)$ & 7.16 & 57.68 & $\begin{array}{l}\text { blend with } \mathrm{CH}_{2} \mathrm{CH}^{13} \mathrm{CN} \text { and } \\
\mathrm{CH}_{3} \mathrm{OH}, v=0\end{array}$ \\
\hline 254 & $25_{7,18}-24_{7,17}$ & 227079.847 & 50 & 191 & 153 & 96 & 44 & - & - & - & - & - \\
\hline 273 & $25_{2,23}-24_{2,22}$ & 231485.527 & 50 & 138 & 165 & 40 & 45 & 0.30 & $13.98(19)$ & 6.27 & 6.59 & blend with U-line? \\
\hline 292 & $26_{6,21}-25_{6,20}$ & 236269.491 & 60 & 186 & 163 & 37 & 46 & 0.36 & $16.71(18)$ & 8.02 & 14.17 & $\begin{array}{l}\text { partial blend with } \mathrm{t}-\mathrm{C}_{2} \mathrm{H}_{5} \mathrm{OCHO} \\
\text { and } \mathrm{U} \text {-line }\end{array}$ \\
\hline 293 & $26_{6,20}-25_{6,19}$ & 236270.459 & 60 & 186 & 163 & 37 & 46 & - & - & - & - & - \\
\hline 306 & $28_{0,28}-27_{0,27}$ & 244765.968 & 21 & 160 & 186 & 39 & 47 & 0.28 & $9.93(19)$ & 6.62 & 10.35 & $\begin{array}{l}\text { blend with } \mathrm{CH}_{3}{ }^{13} \mathrm{CH}_{2} \mathrm{CN}, v=0 \\
\text { and U-line }\end{array}$ \\
\hline 322 & $27_{6,22}-26_{6,21}$ & 245378.722 & 10 & 197 & 170 & 72 & 48 & 0.35 & $17.29(36)$ & 8.29 & 22.20 & blend with ${ }^{13} \mathrm{CH}_{3} \mathrm{CH}_{2} \mathrm{CN}, v=0$ ? \\
\hline 323 & $27_{6,21}-26_{6,20}$ & 245380.146 & 10 & 197 & 170 & 72 & 48 & - & - & - & - & - \\
\hline 368 & $29_{9,20}-28_{9,19}$ & 263364.923 & 22 & 277 & 174 & 74 & 49 & 0.26 & $6.49(37)$ & 8.51 & 9.17 & $\begin{array}{l}\text { baseline problem?, blend with } \\
\text { U-line }\end{array}$ \\
\hline 369 & $29_{9,21}-28_{9,20}$ & 263364.923 & 22 & 277 & 174 & 74 & 49 & - & - & - & - & - \\
\hline 370 & $29_{10,19}-28_{10,18}$ & 263368.355 & 26 & 300 & 170 & 74 & 49 & - & - & - & - & - \\
\hline 371 & $29_{10,20}-28_{10,19}$ & 263368.355 & 26 & 300 & 170 & 74 & 49 & - & - & - & - & - \\
\hline 384 & $29_{6,24}-28_{6,23}$ & 263604.573 & 12 & 221 & 184 & 74 & 50 & 0.28 & $9.92(36)$ & 8.81 & 14.21 & $\begin{array}{l}\text { baseline problem?, partial blend } \\
\text { with } \mathrm{CH}_{3} \mathrm{CH}_{3} \mathrm{CO}, v_{\mathrm{t}}=1 \text { and } \\
\mathrm{CH}_{3} \mathrm{OCH}_{3}\end{array}$ \\
\hline 385 & $29_{6,23}-28_{6,22}$ & 263607.689 & 12 & 221 & 184 & 74 & 50 & - & - & - & - & - \\
\hline 398 & $29_{4,26}-28_{4,25}$ & 264055.836 & 13 & 197 & 189 & 108 & 51 & 0.22 & $17.67(49)$ & 5.92 & 14.22 & $\begin{array}{l}\text { partial blend with } \mathrm{C}_{2} \mathrm{H}_{5} \mathrm{CN}, v=0 \\
\text { and } \mathrm{CH}_{3} \mathrm{CH}_{3} \mathrm{CO}, v=0\end{array}$ \\
\hline
\end{tabular}

Notes: ${ }^{a}$ Numbering of the observed transitions with $S \mu^{2}>20 \mathrm{D}^{2}$ (see Table 2). ${ }^{b}$ Frequency uncertainty. ${ }^{c}$ Lower energy level in temperature units $\left(E_{\mathrm{l}} / k_{\mathrm{B}}\right){ }^{d}$ Calculated rms noise level in $T_{\mathrm{mb}}$ scale. ${ }^{e}$ Numbering of the observed features. ${ }^{f}$ Peak opacity of the amino acetonitrile modeled feature. ${ }^{g}$ Integrated intensity in $T_{\mathrm{mb}}$ scale for the observed spectrum (Col. 10), the amino acetonitrile model (Col. 11), and the model including all molecules (Col. 12). The uncertainty in Col. 10 is given in parentheses in units of the last digit.

contamination by other molecules in the single-dish spectrum of this transition (see Fig. 4a), we expect the Plateau de Bure (PdBI) interferometric flux to be equal to the single-dish flux. Therefore the flux loss of $45 \%$ appearing in the new table for this transition points to an inaccurate intercalibration of the single-dish and PdBI data on the order of $45 \%$ instead.

To solve this intercalibration problem, we compared our PdBI continuum measurements to the continuum measurements done by Liu \& Snyder (1999) at $85 \mathrm{GHz}$ with the Berkeley-Illinois-Maryland Array (BIMA) with a beam of $1.2^{\prime \prime} \times$ $0.5^{\prime \prime}(H P B W)$ at a position angle of $15^{\circ}$. These authors

1 To compute the continuum fluxes in Belloche et al. (2008), we avoided the spectral ranges with detected line emission that otherwise measured a flux of 4.38 Jy over a vertical box of size $3.2^{\prime \prime} \times 4.8^{\prime \prime}$ containing the 4 sources F1 to F4 in the Sgr B2(M) region. To account for the larger PdBI beam $\left(H P B W=3.4^{\prime \prime} \times 0.8^{\prime \prime}\right.$ with a position angle at $10^{\circ}$ ), we integrated the PdBI emission over a slightly larger box of size $3.2^{\prime \prime} \times 5.8^{\prime \prime}$. After correction for primary beam attenuation, we find an integrated flux of $2.7 \mathrm{Jy}$ when the box is centered on F3, and 3.1 Jy when it is centered $0.7^{\prime \prime}$ to the south of F3. Therefore, the PdBI flux is 30 to $40 \%$ smaller than the BIMA flux. The comparison for the SgrB2(N) region is more difficult to perform because Liu \& Snyder (1999) list

would contaminate the continuum fluxes (by up to $10 \%$ toward Sgr B2(N)). Liu \& Snyder (1999) did the same (S.-Y. Liu, private communication). 
Table 5. Measurements obtained toward Sgr B2(N) with the IRAM Plateau de Bure interferometer at $82 \mathrm{GHz}$.

\begin{tabular}{|c|c|c|c|c|c|c|c|c|c|c|c|c|}
\hline Molecule & $F^{a}$ & $\begin{array}{c}f_{\min }^{b} \\
(\mathrm{MHz}) \\
(3)\end{array}$ & $\begin{array}{c}f_{\max }^{b} \\
(\mathrm{MHz}) \\
(4)\end{array}$ & $\begin{array}{c}\sigma^{c} \\
\text { (Jy/beam } \\
(5)\end{array}$ & $\begin{array}{c}F_{\text {peak }}{ }^{d} \\
\left.\mathrm{~km} \mathrm{~s}^{-1}\right) \\
(6)\end{array}$ & $\begin{array}{c}\Delta \alpha^{d} \\
\left({ }^{\prime \prime}\right) \\
(7)\end{array}$ & $\begin{array}{c}\Delta \delta^{d} \\
\left({ }^{\prime \prime}\right) \\
(8)\end{array}$ & $\begin{array}{c}\theta_{\text {maj }}^{f w h m} d \\
\left({ }^{\prime \prime}\right) \\
(9)\end{array}$ & $\begin{array}{c}\theta_{\min }^{f w h m} d \\
\left({ }^{\prime \prime}\right) \\
(10)\end{array}$ & $\begin{array}{c}\mathrm{PA}^{d} \\
\left({ }^{\circ}\right) \\
(11)\end{array}$ & 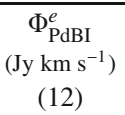 & $\begin{array}{c}\Phi_{30 \mathrm{~m}}^{f} \\
\left(\mathrm{Jy} \mathrm{km} \mathrm{s}^{-1}\right) \\
(13)\end{array}$ \\
\hline AAN & $\mathrm{F} 2$ & 81700.21 & 81703.33 & 0.09 & 0.68 & $-1.60 \pm 0.05$ & $0.30 \pm 0.22$ & $3.9 \pm 0.4$ & $2.00 \pm 0.10$ & $20.5 \pm 0.1$ & 1.76 & 4.10 \\
\hline AAN & F3 & 81708.02 & 81712.08 & 0.10 & 0.68 & $-1.25 \pm 0.06$ & $0.02 \pm 0.24$ & $3.8 \pm 0.5$ & $1.39 \pm 0.12$ & $10.1 \pm 0.0$ & 1.24 & 2.49 \\
\hline AAN & F4 & 81732.71 & 81734.90 & 0.06 & 0.44 & $-1.70 \pm 0.06$ & $0.35 \pm 0.24$ & $3.6 \pm 0.5$ & $1.54 \pm 0.12$ & $14.0 \pm 0.0$ & 0.86 & 1.38 \\
\hline AAN & F5 & 81754.90 & 81757.40 & 0.06 & 0.24 & $-1.52 \pm 0.10$ & $0.03 \pm 0.44$ & $3.2 \pm 0.9$ & $1.20 \pm 0.21$ & $12.5 \pm 1.1$ & 0.30 & 1.63 \\
\hline AAN & F6 & 82223.46 & 82226.27 & 0.06 & 0.43 & $-1.43 \pm 0.06$ & $0.28 \pm 0.24$ & $3.5 \pm 0.5$ & $1.54 \pm 0.11$ & $6.0 \pm 0.4$ & 0.79 & 1.37 \\
\hline Reference & & 81704.27 & 81707.08 & 0.07 & $\ldots$ & $\ldots$ & $\ldots$ & $\ldots$ & $\ldots$ & $\ldots$ & $\ldots$ & $\ldots$ \\
\hline $\mathrm{C}_{2} \mathrm{H}_{5} \mathrm{CN}$ & $\mathrm{HV}$ & 81741.77 & 81744.90 & 0.11 & 2.05 & $-1.64 \pm 0.02$ & $5.58 \pm 0.09$ & $3.8 \pm 0.2$ & $1.50 \pm 0.04$ & $5.7 \pm 0.0$ & 4.07 & 9.03 \\
\hline $\mathrm{C}_{2} \mathrm{H}_{5} \mathrm{CN}$ & LV & 81745.21 & 81749.27 & 0.15 & 2.82 & $-1.74 \pm 0.02$ & $0.46 \pm 0.09$ & $3.8 \pm 0.2$ & $2.87 \pm 0.04$ & $13.7 \pm 0.0$ & 10.43 & 18.32 \\
\hline \multicolumn{2}{|c|}{$\mathrm{HC}^{13} \mathrm{CCN}_{v_{7}}=1$} & 81726.15 & 81728.96 & 0.09 & 2.20 & $-1.35 \pm 0.02$ & $0.60 \pm 0.07$ & $3.7 \pm 0.1$ & $1.68 \pm 0.03$ & $12.6 \pm 0.0$ & 4.98 & 6.81 \\
\hline \multicolumn{2}{|l|}{$\mathrm{HC}_{3} \mathrm{~N} v_{4}=1$} & 81767.71 & 81771.15 & 0.10 & 2.14 & $-1.43 \pm 0.02$ & $0.28 \pm 0.08$ & $3.6 \pm 0.2$ & $1.35 \pm 0.04$ & $9.9 \pm 0.0$ & 3.78 & 5.45 \\
\hline \multirow[t]{2}{*}{$\mathrm{HC}_{3} \mathrm{~N} v_{7}=1^{g}$} & $\mathrm{HV}$ & 82196.27 & 82198.77 & 0.25 & 6.17 & $-2.16 \pm 0.02$ & $0.69 \pm 0.07$ & $4.0 \pm 0.1$ & $1.84 \pm 0.03$ & $16.2 \pm 22.5$ & 16.05 & 33.63 \\
\hline & & & & & 3.36 & $-1.50 \pm 0.03$ & $5.25 \pm 0.12$ & $4.0 \pm 0.2$ & $1.36 \pm 0.06$ & $5.5 \pm 22.5$ & 5.35 & \\
\hline $\mathrm{HC}_{3} \mathrm{~N} v_{7}=1$ & LV & 82199.40 & 82201.58 & 0.36 & 9.06 & $-1.67 \pm 0.02$ & $0.42 \pm 0.07$ & $3.7 \pm 0.1$ & $2.50 \pm 0.03$ & $10.2 \pm 22.5$ & 31.04 & 47.15 \\
\hline $\mathrm{HC}_{3} \mathrm{~N} v_{7}=1$ & BW & 82202.52 & 82203.77 & 0.12 & 3.37 & $-0.71 \pm 0.01$ & $0.24 \pm 0.06$ & $3.1 \pm 0.1$ & $2.77 \pm 0.03$ & $45.0 \pm 0.0$ & 11.75 & 17.44 \\
\hline $\mathrm{CH}_{3} \mathrm{OCHO}$ & & 82242.21 & 82245.33 & 0.10 & 0.67 & $-2.83 \pm 0.06$ & $1.23 \pm 0.26$ & $4.8 \pm 0.5$ & $2.58 \pm 0.12$ & $9.5 \pm 22.5$ & 2.83 & 9.32 \\
\hline
\end{tabular}

${ }^{a}$ Feature numbered like in Col. 8 of Table 3 for amino acetonitrile (AAN). HV and LV mean "high" and "low" velocity components, respectively, and BW means blueshifted linewing. ${ }^{b}$ Frequency range over which the intensity was integrated. ${ }^{c}$ Noise level in the integrated intensity map shown in Fig. 5. ${ }^{d}$ Peak flux, offsets in right ascension and declination with respect to the reference position of Fig. 5, major and minor diameters $(F W H M)$, and position angle (East from North) derived by fitting an elliptical 2D Gaussian to the integrated intensity map shown in Fig. 5. The uncertainty in Col. 11 is the formal uncertainty given by the fitting routine GAUSS_2D, while the uncertainties correspond to the beam size divided by two times the signal-to-noise ratio in Cols. 7 and 8 and by the signal-to-noise ratio in Cols. 9 and $10 .{ }^{e}$ Flux spatially integrated over the region showing emission in the integrated intensity map of Fig. $5 .{ }^{f}$ Integrated flux of the $30 \mathrm{~m}$ spectrum computed over the frequency range given in Cols. 3 and $4 .{ }^{g}$ The two emission peaks of Fig. 5k were fitted separately.

Table 7. Measurements obtained toward Sgr B2(N) with the Australia Telescope Compact Array at $91 \mathrm{GHz}$.

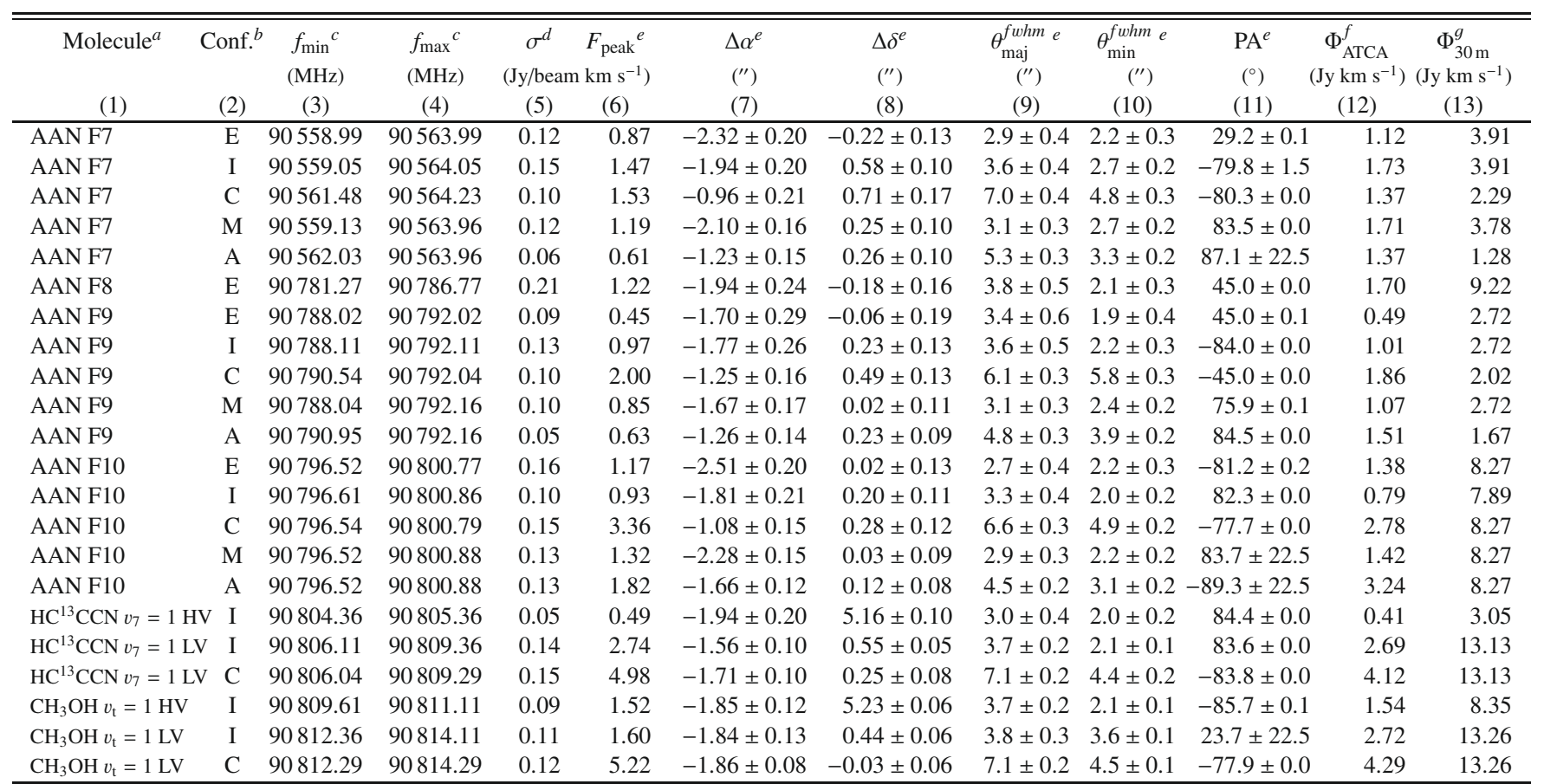

${ }^{a}$ For amino acetonitrile (AAN), we give the feature number like in Col. 8 of Table 3. For the other molecules, HV and LV mean high and low velocity component, respectively. ${ }^{b}$ Interferometer configuration: E: extended (H 214), I: intermediate (H 168), C: compact (H 75), M: mixed (H $214+\mathrm{H} 168)$, A: all $(\mathrm{H} 214+\mathrm{H} 168+\mathrm{H} 75) .{ }^{c}$ Frequency range over which the intensity was integrated. ${ }^{d}$ Noise level in the integrated intensity map shown in Fig. 7. ${ }^{e}$ Peak flux, offsets in right ascension and declination with respect to the reference position of Fig. 7, major and minor diameters $(F W H M)$, and position angle (East from North) derived by fitting an elliptical 2D Gaussian to the integrated intensity map shown in Fig. 7. The uncertainty in Col. 11 is the formal uncertainty given by the fitting routine GAUSS_2D, while the uncertainties correspond to the beam size divided by two times the signal-to-noise ratio in Cols. 7 and 8 and by the signal-to-noise ratio in Cols. 9 and $10 .{ }^{f}$ Flux spatially integrated over the region showing emission in the integrated intensity map of Fig. $7 .{ }^{g}$ Integrated flux of the $30 \mathrm{~m}$ spectrum computed over the frequency range given in Cols. 3 and 4. 
fluxes integrated over boxes smaller than the PdBI beam. They found a flux of $0.90 \mathrm{Jy}$ over a box of size $2.8^{\prime \prime} \times 3.2^{\prime \prime}$ centered on K2. An integration over a larger box of size $2.8^{\prime \prime} \times 4.5^{\prime \prime}$ to account for the larger PdBI beam yields a PdBI flux of $0.72 \mathrm{Jy}$, i.e. $\sim 20 \%$ smaller than the BIMA flux. However, the PdBI flux is contaminated by emission from $\mathrm{K} 3$ and the actual PdBI flux for $\mathrm{K} 2$ is most likely even smaller.

As a result, we think that all PdBI fluxes listed in Table 5 are underestimated by $\sim 30-40 \%$. Since the integration error underestimated the single-dish fluxes by about the same amount, our conclusions about the compact size of the amino acetonitrile emission remain unchanged. On the other hand, the underestimate of the continuum flux led to an underestimate by $~ 30-40 \%$ of the $\mathrm{H}_{2}$ column density, mass, and density in Sect. 3.6, which implies an overestimate of the amino acetonitrile abundance by the same amount. We note, however, that the uncertainty on the quantities derived from the continuum emission is close to a factor of 2, dominated by the uncertainties on the dust properties as we mentioned in Sect. 3.6.

Finally, the single-dish integrated fluxes of Table 7 are all $\sim 30 \%$ larger than before. The ATCA interferometric flux of feature F7 in the full set of configurations (labeled A in Col. 2) now agrees within $10 \%$ with the single-dish flux. This indicates that no emission was filtered out by the interferometer, i.e. that all the emission of amino acetonitrile is from a compact source. This agreement also supports our finding that the fluxes measured with the PdBI were too low, while the calibration of the single-dish spectra should be correct.

As a conclusion, the errors reported here do not change the conclusions we draw about the emission of amino acetonitrile in Sgr B2(N). Only the abundance may have been overestimated by $\sim 30-40 \%$, which is still within its uncertainty.

Acknowledgements. We thank Sheng-Yuan Liu for providing very useful information about the way the flux integration was performed on the BIMA data, and Roberto Neri for helpful discussion about the calibration of the PdBI data.

\section{References}

Belloche, A., Menten, K. M., Comito, C., et al. 2008, A\&A, 482, 179 Liu, S.-Y., \& Snyder, L. E. 1999, ApJ, 523, 683 